OPEN ACCESS

Edited by: Cameron Bass,

Duke University, USA

Reviewed by:

Linda Noble,

University of California San Francisco,

USA

Firas H. Kobeissy,

University of Florida, USA

${ }^{*}$ Correspondence:

W. Alan C. Mutch

wacmutch@shaw.ca

Specialty section: This article was submitted to Neurotrauma,

a section of the journa

Frontiers in Neurology

Received: 15 February 2016

Accepted: 11 April 2016

Published: 29 April 2016

Citation:

Ellis MJ, Ryner LN, Sobczyk O, Fierstra J, Mikulis DJ, Fisher JA,

Duffin J and Mutch WAC (2016)

Neuroimaging Assessment of

Cerebrovascular Reactivity in

Concussion: Current Concepts,

Methodological Considerations, and

Review of the Literature.

Front. Neurol. 7:61

doi: 10.3389/fneur.2016.00061

\section{Neuroimaging Assessment of Cerebrovascular Reactivity in Concussion: Current Concepts, Methodological Considerations, and Review of the Literature}

\author{
Michael J. Ellis ${ }^{1,2,3,4,5,6,7}$, Lawrence N. Ryner,8,9, Olivia Sobczyk ${ }^{10}$, Jorn Fierstra ${ }^{11}$, \\ David J. Mikulis ${ }^{12,13,14}$, Joseph A. Fisher ${ }^{13,14,15}$, James Duffin ${ }^{13,14,16}$ and \\ W. Alan C. Mutch $6,7,9,17 *$
}

${ }^{1}$ Department of Surgery, University of Manitoba, Winnipeg, MB, Canada, ${ }^{2}$ Department of Pediatrics and Child Health, University of Manitoba, Winnipeg, MB, Canada, ${ }^{3}$ Section of Neurosurgery, University of Manitoba, Winnipeg, MB, Canada, ${ }^{4}$ Pan Am Concussion Program, University of Manitoba, Winnipeg, MB, Canada, ${ }^{5}$ Childrens Hospital Research Institute of Manitoba, University of Manitoba, Winnipeg, MB, Canada, ${ }^{6}$ Canada North Concussion Network, University of Manitoba, Winnipeg, MB, Canada, ${ }^{7}$ University of Manitoba, Winnipeg, MB, Canada, ${ }^{8}$ Department of Radiology, University of Manitoba, Winnipeg, MB, Canada, ${ }^{9}$ Health Sciences Centre, University of Manitoba, Winnipeg, MB, Canada, ${ }^{10}$ Institute of Medical Sciences, University of Toronto, Toronto, ON, Canada, " Department of Neurosurgery, University Hospital Zurich, Zurich, Switzerland, ${ }^{12}$ Department of Medical Imaging, University of Toronto, Toronto, ON, Canada, ${ }^{13}$ University of Toronto, Toronto, ON, Canada, ${ }^{14}$ University Health Network Cerebrovascular Reactivity Research Group, Toronto, ON, Canada, ${ }^{15}$ Department of Anesthesia, University of Toronto, Toronto, ON, Canada, ${ }^{16}$ Department of Physiology, University of Toronto, Toronto, ON, Canada, ${ }^{17}$ Department of Anesthesia and Perioperative Medicine, University of Manitoba, Winnipeg, MB, Canada

Concussion is a form of traumatic brain injury (TBI) that presents with a wide spectrum of subjective symptoms and few objective clinical findings. Emerging research suggests that one of the processes that may contribute to concussion pathophysiology is dysregulation of cerebral blood flow (CBF) leading to a mismatch between CBF delivery and the metabolic needs of the injured brain. Cerebrovascular reactivity (CVR) is defined as the change in CBF in response to a measured vasoactive stimulus. Several magnetic resonance imaging (MRI) techniques can be used as a surrogate measure of CBF in clinical and laboratory studies. In order to provide an accurate assessment of CVR, these sequences must be combined with a reliable, reproducible vasoactive stimulus that can manipulate CBF. Although CVR imaging currently plays a crucial role in the diagnosis and management of many cerebrovascular diseases, only recently have studies begun to apply this assessment tool in patients with concussion. In order to evaluate the quality, reliability, and relevance of CVR studies in concussion, it is important that clinicians and researchers have a strong foundational understanding of the role of CBF regulation in health, concussion, and more severe forms of TBI, and an awareness of the advantages and limitations of currently available CVR measurement techniques. Accordingly, in this review, we (1) discuss the role of CVR in TBI and concussion, (2) examine methodological considerations for MRI-based measurement of CVR, and (3) provide an overview of published CVR studies in concussion patients.

Keywords: concussion, cerebrovascular reactivity, magnetic resonance imaging, blood oxygen level-dependent imaging, carbon dioxide 


\section{BACKGROUND}

Traumatic brain injury (TBI) continues to be one of the leading causes of death and disability among young people worldwide. The pathophysiology of TBI is characterized by a primary injury that results in structural injury to the brain, followed by a secondary injury that is caused by a combination of inflammation, edema, ischemia, cellular necrosis, and apoptosis (1). Some of these mechanisms are readily detectable on clinical neuroimaging studies in mild, moderate, and severe TBI patients; with the presence of certain features highly correlated with patient outcome $(2,3)$. Although mTBI and concussion are terms that are often used interchangeably to describe patients with similar clinical presentations, concussion is thought to represent a form of mTBI that typically occurs in the absence of structural brain injury (4). Consequently, clinical neuroimaging studies are normal in the vast majority of patients (5), and the clinical manifestations of this condition are proposed to be mediated by neuronal dysfunction and cerebrovascular dysregulation $(6,7)$.

Among the most important factors responsible for maintaining brain function during health and injury is the process of matching cerebral blood flow $(\mathrm{CBF})$ to the metabolic needs of the brain. In the setting of TBI, periods of mismatched CBF can result in secondary brain injury that can impact patient outcomes (8-11). Cerebrovascular reactivity (CVR), defined as the change in $\mathrm{CBF}$ in response to a vasoactive stimulus, is a means to interrogate the integrity of the effector arm of the demand-supply control of CBF. To measure CVR among patients with cerebrovascular disease including stroke, TBI, and concussion, research studies have combined the use of several advanced non-invasive neuroimaging techniques with several types of vasoactive stimuli. While the neuroimaging techniques used to assess $\mathrm{CBF}$ in these studies are highly reliable and reproducible, the vasoactive stimuli used to manipulate CBF are not (12). Several studies have examined CVR in patients with moderate and severe TBI $(11,13)$, however, only recently have studies examining CVR in patients with concussion become available (14-17). It is therefore important for clinicians and researchers interested in the role of $\mathrm{CBF}$ regulation in the pathophysiology of concussion to be able to evaluate the quality, reliability, and implications of studies performed in this population.

Accordingly, the objectives of the present review were threefold: (1) to discuss the role of cerebrovascular physiology in the health and the injured brain including the concussed brain, (2) to review the role and limitations of MRI-based tools and vasoactive stimuli used to measure CVR, and (3) to provide an overview of published CVR studies in concussion patients.

\section{CEREBRAL BLOOD FLOW REGULATION, CEREBROVASCULAR REACTIVITY, AND TRAUMATIC BRAIN INJURY}

\section{Cerebral Blood Flow Regulation and Cerebrovascular Reactivity}

Although the brain accounts for only $2 \%$ of total body weight, it receives $15-20 \%$ of the cardiac output in humans. In order to maintain the health of this essential organ, the cerebral vasculature must maintain $\mathrm{CBF}$ within precise limits under a wide spectrum of states during rest, activity, and disease. Control of $\mathrm{CBF}$ is achieved primarily by altering the flow resistance within the cerebral blood vessels at the arteriolar level. In a more global sense, $\mathrm{CBF}$ is dependent on the cerebral perfusion pressure (CPP), which is influenced by the mean arterial blood pressure (MAP), the intracranial pressure (ICP), and the resistance of the major arteries. However, on a regional scale, in order to meet the increased energy demands of the brain during activation or disease, the cerebral vasculature must respond appropriately to more localized physiological stimuli, which include the direct action of signaling messengers such as calcium ions, nitric oxide, acetylcholine, vasoactive intestinal polypeptide (VIP), calcitonin generelated peptide (CGRP), substance $\mathrm{P}$, prostaglandins, and other arachadonic acid metabolites released by astrocytes and neurons as well as local factors such as intraluminal hydrostatic pressure, $\mathrm{pH}$, and partial pressure of arterial carbon dioxide $\left(\mathrm{PaCO}_{2}\right)$ and oxygen $\left(\mathrm{PaO}_{2}\right)$ (18) (see Figure 1). Laboratory evidence also suggests that capillary pericytes respond to similar physiological stimuli and play an important role in CBF regulation during neuronal activation and ischemia at the micro-regional level (19). Failure to appropriately match CBF with surges in the metabolic demands of the brain can lead to temporary or permanent alterations in neurological functioning. This strong dependence is accomplished by a process known as neurovascular coupling, whereby increases in neuronal activity, and consequently, the cerebral metabolic rate of oxygen $\left(\mathrm{CMRO}_{2}\right)$ is tightly related to corresponding increases in regional $\mathrm{CBF}$. Modulation of $\mathrm{CBF}$ during neuronal activity requires the coordinated efforts of the neurovascular unit, which includes neurons, astrocytes, pericytes, microglia, and the vascular endothelial and smooth muscle cells (20), and typically results in an "overshoot" in blood flow with respect to $\mathrm{O}_{2}$ demand, thereby delivering more oxygen than is consumed by the active assembly of neurons. It has therefore been postulated that this functional hyperemia is required for the delivery of other substrates such as glucose, or the removal of metabolic waste or heat. Acute or chronic insufficiency in CBF delivery can exist along a continuum resulting in a wide spectrum of disease conditions including transient ischemic attack (TIA), acute ischemic stroke, and vascular dementia (21). One of the ways to assess the functional integrity of this complex regulatory system is to measure the brain's cerebrovascular response to a vasoactive stress or stimulus. A vasoactive stimulus is a stimulus that can be applied to the brain and produces a quantifiable and reliable effect on the cerebral vasculature, usually stimulating vasodilation.

In general, the effect of any vasodilatory stimulus on any vascular bed in the brain is dependent on (1) the magnitude of the vasodilatory stimulus; (2) the difference in vasodilatory reserve of the interrogated vascular bed and that of the beds with which it is perfused in parallel; and (3) the flow reserve of the feeding vessel to both beds (22) (Figure 2A). Therefore, with intact vasodilatory reserves, the application of a global vasodilatory stimulus like hypercapnia, will produce a balanced reduction in vascular tones and a symmetrical increase in CBF in all beds (Figure 2B). However, if the vasodilatory reserve of a particular vascular bed 


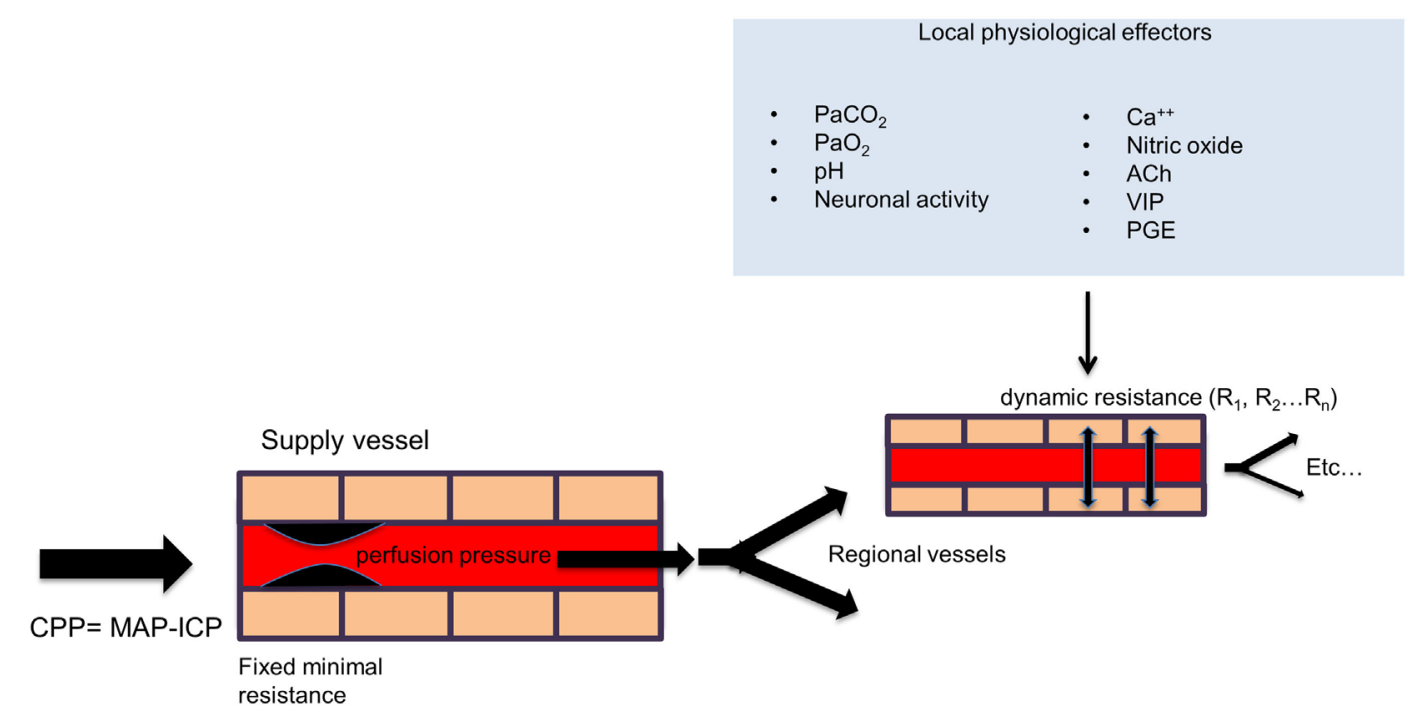

FIGURE 1 | General schema of cerebral perfusion. Intracranial vessels are perfused in parallel in a fractal branching pattern. The net flow in each region is dynamically determined by the net flow resistance of each branch. Under normal conditions, the inflow from major extracranial vessels is not limiting. The flow to each vascular region is controlled by its local factors as shown in the figure. The net effect of regional vascular resistances determines the total cerebral blood flow. However, with a strong global vasodilatory stimulus, the drop in resistance in the collective downstream branches can be reduced to the point where the blood flow in the larger supply vessels is limiting ("fixed minimal resistance" in supply vessel in the figure). Abbreviation: CPP, cerebral perfusion pressure; MAP, mean arterial pressure; ICP, intracranial pressure; $\mathrm{PaCO}_{2}$, arterial partial pressure of carbon dioxide; $\mathrm{PaO}_{2}$, arterial partial pressure of oxygen; Ca ${ }^{++}$, calcium ions; $\mathrm{ACh}$, acetylcholine; VIP, vasoactive intestinal polypeptide; PGE, prostaglandins.

is compromised, the application of a vasodilatory stimulus will result in an asymmetrical vasodilatory response (Figure 2C). When there is adequate inflow reserve, the changes in flow to each vascular bed follows its change in resistance. When the total flow demand of the beds exceeds the inflow capability, a condition to which the brain vasculature is uniquely susceptible (23), there is a redistribution of blood flow in favor of the vascular bed with better vasodilatory reserve. In severe cases where vasodilatory reserve is diminished or abolished in a vascular bed, the flow becomes completely dependent on the perfusion pressure and on the relative changes in resistance of the vascular beds with intact vasodilatory reserve that are perfused in parallel. Therefore, even in the setting of normal global mean CBF, regional impairments in the control of vascular resistance can lead to regional hypo- and hyperperfusion (see Figure 2C). This mechanism can apply to vessels that range in scale from capillaries to cerebral arteries and even extracranial arteries (24). Such cerebrovascular dysregulation may be unmasked by applying a global vasodilatory stimulus to the cerebral vasculature and observing changes in regional $\mathrm{CBF}$.

While these concepts are easy to understand, they are somewhat difficult to apply in practice. Indeed, CVR varies considerably even among healthy subjects (25) and within a healthy subject over time (26). That CVR normally varies considerably between brain regions and between white matter and gray matter makes accurate assessment and interpretation of CVR studies challenging (27-30). Within these regions, sustained hypoperfusion or the inability to adequately respond to metabolic demand surges (as detected by CVR) can lead to local brain dysfunction
(31) and structural degeneration (32). Furthermore, impairments in CVR have been associated with numerous cerebrovascular diseases such as intra- and extracranial steno-occlusive disease (33-37), arteriovenous malformations (38), cerebral proliferative angiopathy (39), and cerebral vasospasm following aneurysmal subarachnoid hemorrhage (40) and are strongly predictive of the development of ischemic stroke (41-43). Given the enormous increases in energy requirements associated with TBI, the adequacy of the cerebrovascular response to match CBF supply to the metabolic demands of the injured brain is likely an important consideration impacting clinical deficits and injury recovery.

\section{Cerebrovascular Disturbances in TBI}

The pathophysiology of TBI is characterized by primary and secondary brain injury. Primary brain injury refers to the direct biomechanical disruption of brain tissue that occurs at the time of initial impact and which can range from focal injuries such as parenchymal contusions and subdural hematoma to more global injuries such as diffuse axonal injury (DAI). While these processes account for a significant proportion of the morbidity and mortality associated with moderate and severe TBI, these processes are not thought to play a prominent role in the pathophysiology of concussion (4). On the other hand, secondary brain injury is characterized by a combination of cellular, metabolic, and inflammatory processes that can result in further tissue edema, injury, and associated neurological deterioration $(44,45)$. One of the processes that play an important role in secondary brain injury is ischemia. Pathological features of ischemic injury are present in more than $90 \%$ of autopsy specimens from patients 

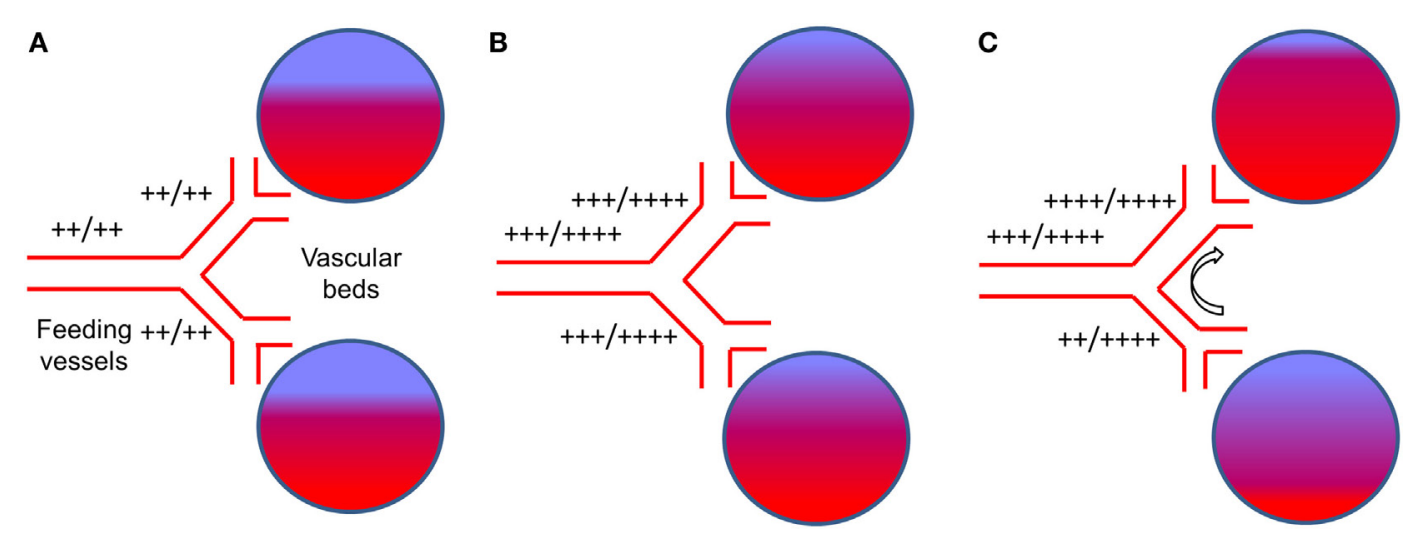

FIGURE 2 | The effect of a global vasodilatory stimulus on regional blood flow with normal vasculature and with impaired regional vascular response. (A) The normal state at normocapnia. The extent of red color in the vascular beds represents actual blood flow and blue color represents potential blood flow. " $++/++$ " beside vessels represents normal blood flow at rest $(++)$ compared to the flow demand $(++)$. This would be the case for normal vasculature and for vasculature that has branches with reduced vasodilatory capacity. (B) With normal vasculature, hypercapnia stimulates increase blood demand by the vascular beds. The vasodilatory demand of the vascular beds combined exceed that of the main feeding vessel (23), which is limiting, i.e., their flow (+++) does not meet demand $(++++)$. However, the dilatory response capability of each feeding vessel is symmetrical and so is their flow. (C) In the presence of a dysfunctional vessel, a hypercapnic stimulus results in the same demand in the healthy and dysfunctional vessel (i.e., ++++). There is a strong vasodilation in the healthy (upper) branch and a weaker vasodilation in the dysfunctional (lower) branch. The inflow from the main vessel is still limiting (i.e., $+++/++++)$. The direct competition for flow between the vascular beds results in an increased proportion of the flow through the normal vessel (++++) at the expense of the dysfunctional vessel [flow reduced from +++ in $(\mathbf{B})$, to ++ ]. This is referred to as vascular 'steal'.

with fatal TBI $(46,47)$. Experimental evidence suggests that CBF can fall to $50 \%$ of baseline levels in patients with severe TBI (48) and can remain persistently low for weeks to years after injury (49). That this decrease in CBF delivery occurs in the setting of increased metabolic need suggests that the pathophysiology of TBI may have a neurovascular component. On a smaller scale, imbalances in neuronal activity cellular ion transport, increased excitatory neurotransmitter release, and impaired glucose metabolism coupled with insufficient CBF delivery are proposed to account for the "neurometabolic energy crisis" that characterizes acute concussion $(6,7)$. In addition to concussion and more severe forms of TBI that can occur in sports- and nonsports-related activities, blast-related TBI is a unique form of TBI that presents with similar symptoms and is also characterized by diffuse primary and secondary brain injury processes (50). In particular, animal model evidence suggests that blast-related TBI is associated with time-dependent alterations in many serum proteins responsible for brain cellular metabolic and cerebrovascular processes (51).

Neuroimaging studies in patients with moderate and severe TBI have provided important insights into secondary brain injury and suggest that alterations in CBF and CVR have an important impact on patient outcomes. Bouma et al. (8) found significant correlations between low $\mathrm{CBF}$ and clinical motor scores and functional outcomes following severe TBI using Xe-CT. Onethird of patients exceeded the ischemic stroke CBF threshold of $<18 \mathrm{ml} / 100 \mathrm{~g} / \mathrm{min}$ within $6 \mathrm{~h}$ of injury, which was associated with higher mortality rates and worse outcomes among survivors. Using positron emission tomography (PET) measurements, Coles et al. (9) found that ischemic brain volumes were significantly higher in severe TBI than controls and correlated with poor 6-month patient outcomes. Wintermark et al. (10) using perfusion-CT found that low regional CBF following TBI was associated with worse 3-month outcomes. Finally, Adelson et al. (11), using Xe-CT showed that mean CBF $<20 \mathrm{ml} / 100 \mathrm{~g} /$ min and $\mathrm{CVR}<2 \% / \mathrm{mm} \mathrm{Hg} \mathrm{PaCO}_{2}$ within 2 days of injury were associated with unfavorable outcomes. More recently, Maugans et al. (52) demonstrated impaired mean resting CBF in the acute phase of pediatric sports-related concussion (SRC) that persisted at 1 month despite normalization of neurocognitive testing scores. Bartnik-Olson et al. (53) observed reductions in CBF and cerebral blood volume in the thalamus of pediatric SRC patients compared to normal controls. Meier et al. (54) detected regional abnormalities in resting CBF among collegiate SRC patients during acute injury that were normalized 1 month later. In this latter study, regional $\mathrm{CBF}$ within two regions of interest correlated with scores on a neuropsychiatric symptom inventory. Finally, Wang et al. (55) detected regional reductions in CBF within $24 \mathrm{~h}$ of injury in collegiate athletes that persisted at day 8 post-injury despite normalization of clinical and neurocognitive testing scores (for summary, see Table $\mathbf{1}$ ).

Overall, evidence from CVR studies in severe TBI and preliminary studies examining resting CBF in SRC patients supports the conceptual understanding of concussion as characterized by persistent cerebrovascular dysregulation and provides a rationale for examining the role of CVR in patients with concussion.

\section{METHODOLOGICAL CONSIDERATIONS IN MRI-BASED MEASUREMENT OF CVR}

Over the past 20 years, advances in neuroimaging technology have permitted clinicians and researchers to accurately assess cerebrovascular physiology using a number of techniques. At present, 
TABLE 1 | Overview of studies examining resting cerebral blood flow in concussion.

\begin{tabular}{|c|c|c|c|c|c|}
\hline Reference & Study population & Methodology & $\begin{array}{l}\text { Clinical concussion } \\
\text { measures }\end{array}$ & $\begin{array}{l}\text { Neuroimaging } \\
\text { sequence }\end{array}$ & Results \\
\hline (52) & $\begin{array}{l}12 \text { SRC patients (age } \\
11-15 \text { years) imaged } \\
<72 \mathrm{~h}, 14 \text { days, and } \\
30 \text { days or more post- } \\
\text { injury and } 12 \text { control } \\
\text { subjects }\end{array}$ & Longitudinal & ImPACT & ASL and DTI MRI & $\begin{array}{l}\text { Alterations in mean resting } \mathrm{CBF} \text { (predominantly reduced } \\
\mathrm{CBF} \text { ) in the acute phase of SRC that persisted at } 1 \text { month } \\
\text { despite normalization of neurocognitive testing scores }\end{array}$ \\
\hline (53) & $\begin{array}{l}15 \text { PCS patients (age } \\
8-17 \text { years) imaged } \\
\text { 3-12 months post-injury } \\
\text { and } 15 \text { control subjects }\end{array}$ & Cross-sectional & $\begin{array}{l}\text { Self-reported } \\
\text { symptoms }\end{array}$ & $\begin{array}{l}\text { Perfusion-weighted } \\
\text { and DTI MRI, } \\
\text { MRI-spectroscopy }\end{array}$ & $\begin{array}{l}\text { PCS group demonstrated reduced CBF and CBV in the } \\
\text { bilateral thalami compared to the control group. No other } \\
\text { significant differences in other brain regions }\end{array}$ \\
\hline (54) & $\begin{array}{l}15 \text { SRC patients (mean } \\
\text { age }=20.57 \text { years) } \\
\text { imaged at } 0-3 \text { days, } \\
6-13 \text { days, and } \\
25-44 \text { days post-injury } \\
\text { and } 27 \text { control subjects }\end{array}$ & Longitudinal & $\begin{array}{l}\text { Hamilton Depression } \\
\text { and Anxiety rating } \\
\text { scales, ANAM }\end{array}$ & ASL MRI & $\begin{array}{l}\text { Reduced CBF in the right dorsal midinsular cortex (dmIC) } \\
\text { and superior temporal sulcus in SRC group during acute } \\
\text { phase of injury that were similar to the control group at } \\
1 \text { month post-injury } \\
\text { Regional blood flow within the dmIC was inversely related } \\
\text { to the magnitude of initial psychiatric symptoms }\end{array}$ \\
\hline (55) & $\begin{array}{l}18 \mathrm{SRC} \text { patients (mean } \\
\text { age }=17.8 \text { years) } \\
\text { imaged within } 24 \mathrm{~h} \text { and } \\
8 \text { days post-injury and } \\
19 \text { control subjects }\end{array}$ & Longitudinal & $\begin{array}{l}\text { Sport Concussion } \\
\text { Assessment Tool- } \\
\text { 3, Standardized } \\
\text { Assessment of } \\
\text { Concussion, Balance } \\
\text { Error Scoring System, } \\
\text { ANAM, ImPACT }\end{array}$ & pCASL MRI & $\begin{array}{l}\text { At } 24 \text { h post-injury reduced CBF was observed in the } \\
\text { SRC group within the right supplementary motor area } \\
\text { and pre-supplementary motor areas compared to the } \\
\text { control group. At } 8 \text { days post-injury reduced CBF was } \\
\text { observed in diffuse cortical gray matter and thalamus in } \\
\text { the SRC group compared to the control group despite } \\
\text { normalization of clinical concussion measures }\end{array}$ \\
\hline
\end{tabular}

TBI, traumatic brain injury; SRC, sports-related concussion; PCS, postconcussion syndrome; ImPACT, immediate post-concussion assessment and cognitive testing; ASL, arterial spin labeling; DTI, diffusion tensor imaging; CBF, cerebral blood flow; ANAM, automated neuropsychological assessment metrics.

the gold standard for measuring cerebrovascular parameters such as $\mathrm{CBF}$ and $\mathrm{CMRO}_{2}$ is PET while single photon emission computerized tomography (SPECT) provides comparable assessment of CBF. As previously mentioned, XeCT has also been used to measure CVR in previous studies. Unfortunately, such approaches employ radiation exposure, intravenous administration of contrast agents, and occasionally general anesthesia and intubation with mechanical ventilation to achieve inspired gas control, making these inappropriate techniques for the longitudinal assessment of patients with concussion, especially children and adolescents. Transcranial Doppler ultrasonography has also been used to measure CVR in normal patients and in various cerebrovascular conditions such as concussion (56). It is readily available, inexpensive, and non-invasive; however its poor spatial resolution for CVR, CBF, and tissue perfusion limit its value.

Because CVR represents the unit change in $\mathrm{CBF}$ per unit change in a vasoactive stimulus, it is important that both of these variables are measured accurately and applied consistently across study subjects. Failure to do so can lead to differences in CVR measurements between subjects and subject groups that are due to the methodological limitations and variability in techniques and not the disease processes under investigation. Therefore, in order to provide an accurate, reliable, and reproducible MRI-based measure of CVR in humans and any patient population an assessment tool must combine three essential elements: (1) an MRI sequence that can be applied to the entire brain and provide an accurate assessment of CBF or its surrogate at high spatial resolution; (2) a standardized vasoactive stimulus that can provide a measurable and reproducible effect on the cerebral vasculature; and (3) a standardized method of CVR data analysis that allows comparisons between subject groups, and in individual subjects over time.

\section{Cerebral Blood Flow Assessment}

Among the most common non-invasive MRI sequences that are used to assess CBF are blood oxygen level-dependent (BOLD) and arterial spin labeling (ASL). BOLD-level MRI is a technique that exploits the unique MRI signal characteristics of hemoglobin across different oxygenated states within the brain. Simply put, changes in $\mathrm{CBF}$ within a specific brain region are associated with changes in the concentration of hemoglobin that transitions between oxygenated and de-oxygenated states within that region. This transition is associated with a quantifiable change in the ratio between oxyhemoglobin $\left(\mathrm{HbO}_{2}\right)$ and deoxyhemoglobin $(\mathrm{HHb})$. $\mathrm{HHb}$ is paramagnetic and degrades the BOLD signal in the vessels and surrounding brain tissue. Under normal circumstances, neuronal activation leads to an increase in CBF where the increased delivery of oxygen often exceeds the oxygen consumption of the surrounding tissue (57) resulting in an increase in the $\mathrm{HbO}_{2} / \mathrm{HHb}$ ratio and a resultant increase in the BOLD signal. Within a limited range of $\mathrm{CBF}$, changes in BOLD signal are proportional to reductions in the concentration of $\mathrm{HHb}$. The relationship between the BOLD signal and CBF under a constant $\mathrm{CMRO}_{2}$ is fairly linear for $\mathrm{CBF}$ increases up to $50 \%$ (58), and since CBF changes induced during CVR studies are within this magnitude, the BOLD signal can be used as a surrogate for $\mathrm{CBF}$ measurement. However, emerging evidence suggests that the hemodynamic response to neuronal activity is a product of a relationship between neuronal energy consumption and signaling processes (59-61), CBF, 
$\mathrm{CMRO}_{2}, \mathrm{CBV}$, and hemoglobin concentration. Thus, under conditions when brain tissue $\mathrm{O}_{2}$ consumption increases beyond normal levels (i.e., during injury), this relationship becomes more tenuous.

In contrast, ASL is a technique that can provide a direct measurement of CBF within brain tissue. To accomplish this, a radiofrequency pulse is applied to label incoming blood water protons to invert proton magnetization. These labeled protons can be measured once the blood reaches the perfusion bed of the target tissue. The balance between labeled intravascular water and free water in the tissue is proportionate to the CBF, which can then be modeled using post-processing algorithms to generate $\mathrm{CBF}$ in units of $\mathrm{ml} / 100 \mathrm{~g}$ tissue/min (62). This technique can be used to measure global and regional resting CBF but must be interpreted with caution in the setting of atherosclerotic steno-occlusive disease where damaged or blocked vessels can increase transit times, leading to delays in arrival of labeled protons and thereby producing errors in the CBF measurement. In fact, there are no publications showing the efficacy of current ASL techniques for measuring CBF quantitatively in patients with advanced cerebrovascular disease. At present, PET remains the only accurate method for doing so but has lower spatial resolution. Nevertheless, attempts to combine ASL with a vasoactive stimulus to measure CVR have shown efficacy when assessing limited brain regions (i.e., nonwhole brain coverage) (35). Future refinements of the method (delay insensitive whole brain ASL) may eventually lead to replacement of BOLD for measurement of CVR in advanced cerebrovascular disease.

Taken together, both BOLD and ASL techniques provide accurate, reliable, and reproducible measures of whole brain and regional $\mathrm{CBF}$ and can provide similar measures of CVR if paired with a reliable and reproducible vasoactive stimulus.

\section{Vasoactive Stimuli}

In order to provide an accurate measure of CVR between study groups, it is important that assessment tools ensure that subjects are exposed to the same magnitude and duration of vasoactive stimulus (12). This aim can only be accomplished if the vasoactive stimulus produces a quantifiable and reliable effect on the cerebral vasculature and if the magnitude of the stimulus can be measured at the time of administration. In the case of longitudinal studies, it is paramount that the same magnitude of vasoactive stimulus is applied and measured on repeat examinations. If the clinician or researcher cannot confirm that the same stimulus is applied across subjects and during serial assessments within subjects, it is impossible to conclude whether the differences in CVR are a consequence of changes in the disease process or a function of the variability in the study technique $(25,26)$.

The main vasoactive stimuli that have been used to manipulate $\mathrm{CBF}$ in CVR studies in stroke and TBI populations fall into two categories: (1) injection of an exogenous vasodilatory agent (i.e., acetazolamide); (2) modulation of $\mathrm{PaCO}_{2}$ via inhaled $\mathrm{CO}_{2}$, breath-holding, or prospective end-tidal targeting. Each of these techniques has its own advantages and limitations that must be considered in the setting of the patient population under study.
Acetazolamide is a carbonic anhydrase inhibitor that produces a vasodilatory effect on the cerebral vasculature by inducing an intracellular and extracellular acidosis. Since its first introduction to measure CVR in $1986(63,64)$, it has been combined with several neuroimaging techniques to measure CVR in various cerebrovascular disease populations. While intravenous administration of acetzolamide is safe, its side effect profile and effect on CBF are unpredictable. In one study, $63 \%$ of subjects administered low dose acetazolamide developed symptoms such as headaches, nausea, dizziness, weakness or numbness of the extremities, and fatigue lasting $0.5-72 \mathrm{~h}$ that occurred more frequently in younger patients and females (65). At higher doses, these side effects can be more severe requiring treatment, which in many cases results in prompt termination of the study $(66,67)$. While these symptoms may be tolerated in normal control subjects and those with asymptomatic cerebrovascular disease, patients with acute SRC and PCS typically have many of these symptoms at rest making prolonged exacerbation of these symptoms undesirable. The need for intravenous access is another disadvantage of this technique, making it less appropriate for use in children and adolescents. However, the most significant disadvantage of this technique for use in CVR studies is the unpredictable pharmacokinetics of acetazolamide administration and its resultant effects on CBF. Serum concentrations following intravenous administration have been found to vary widely between individuals preventing a reliable stimulus-response relationship to be established (12). Even at supramaximal doses, acetazolamide does not produce a maximal CBF response in many normal subjects $(66,67)$ and can continue to be modulated by the effects of respiratory rate and blood pressure during neuroimaging acquisition. Although some studies have demonstrated that CVR measurements using acetazolamide are comparable to studies using inspired $\mathrm{CO}_{2}$ and breath-holding techniques (68-70), they are not reliably so, and there are other techniques that provide a better tolerated and more reliable vasoactive stimulus.

$\mathrm{PaCO}_{2}$ is the most potent stimulus for cerebral vasculature vasodilation whereby each millimeter of mercury unit increase is associated by a $2-15 \%$ increase in $\operatorname{CBF}(71,72)$. Increases in $\mathrm{P}_{\mathrm{ET}} \mathrm{CO}_{2}$ levels of 5-10 $\mathrm{mmHg}$ are commonly experienced among children and adults during sleep and physical activities and are well tolerated during studies targeting these levels (73). While administering a fixed "dose" of inspired $\mathrm{CO}_{2}$ makes intuitive sense, the $\mathrm{PaCO}_{2}$ is not simply a function of the inspired $\mathrm{PCO}_{2}$. Rather, it is also a function of the ventilatory response, which is a function of the individual $\mathrm{CO}_{2}$ sensitivity that varies between subjects, as will the $\mathrm{PETCO}_{2}$ in response to fixed inspired $\mathrm{PCO}_{2}$. Since the subject's ventilatory response cannot be restrained or predicted, neither can the $\mathrm{PaCO}_{2}$ (74). Furthermore, the $\mathrm{PaCO}_{2}$ (the actual stimulus) is not reliably known as it is loosely related to $\mathrm{PETCO}_{2}$ (what is measured), thus reducing the signal-to-noise ratio (SNR) for CVR measurement. Hyperventilation during a hypercapnic challenge can also lead to increases in end-tidal $\mathrm{O}_{2}\left(\mathrm{P}_{\mathrm{ET}} \mathrm{O}_{2}\right)$ and $\mathrm{PaO}_{2}$ (up to $15 \mathrm{mmHg}$ ) (75), which can result in an increase in BOLD signal that is independent of the $\mathrm{PaCO}_{2}$ changes leading to falsely elevated CVR values (76). Furthermore, the $\mathrm{BP}$ response to $\mathrm{CO}_{2}$ can also vary considerably among normal control subjects and can act as an additional confounding variable for accurate CVR measurement (29). Altogether, there are numerous individual differences that 
influence CVR measurement; factors that are difficult to account for unless $\mathrm{PETCO}_{2} \mathrm{PeTO}_{2}, \mathrm{HR}, \mathrm{BP}$, and ventilatory rate are rigorously and frequently measured during image acquisition.

For those studies that use block design $\mathrm{CO}_{2}$ breathing protocols, the rates of change of $\mathrm{PaCO}_{2}$ and $\mathrm{PaO}_{2}$, are unpredictable, other than knowing that no plateau is likely to be achieved within the duration of a protocol. Because CVR represents the change in CBF per unit change in $\mathrm{CO}_{2}$, some studies have used $\mathrm{PETCO}_{2}$ as a surrogate for $\mathrm{PaCO}_{2}$. However, the differences between $\mathrm{PETCO}_{2}$ and $\mathrm{PaCO}_{2}$ depend on a number of factors including age, exercise, body composition, and other features of respiratory physiology. Even under conditions of accurate and frequent $\mathrm{PeTCO}_{2}$ and $\mathrm{PETO}_{2}$ measurement, $\mathrm{CO}_{2}$ inhalation generates an imprecise vasoactive stimulus and therefore an imprecise measure of CVR.

Another technique that has been long used to generate a hypercapnic stimulus in CVR studies is breath holding. The primary advantage of this technique is it requires no breathing circuits or equipment to administer $\mathrm{CO}_{2}$. A simplifying assumption is that breath-holding results in a linear rise in $\mathrm{PaCO}_{2}$ with time. In this way, the magnitude of the vasoactive stimulus is conceptually quantified according to the duration of breath-holding. However, the rise of $\mathrm{PaCO}_{2}$ with time is not linear; the tolerance for duration of breath-holding varies widely between people (68); the $\mathrm{PaO}_{2}$ drops precipitously with time; and the $\mathrm{PeTCO}_{2}$ at the end of breath-holding is not necessarily equal to the $\mathrm{PaCO}_{2}$. Consequently, there is no consensus on how breath-holding data should be analyzed as there is no stimulus information (no knowledge of $\mathrm{PaCO}_{2}$ levels) and selection of an appropriate regressor to regress against the BOLD data for generation of global CVR maps is problematic. Finally, as breath-holding relies on repeated sustained Valsalva maneuvers, it is undesirable for use in patients with acute SRC and PCS who, in our clinical experience, frequently report an exacerbation of their concussion symptoms with this type of exertion.

Among currently available techniques, only automated computer controlled gas blenders have proved capable of delivering a precise, highly controlled, and measurable hypercapnic stimulus for CVR studies $(75,77)$. These techniques are capable of precise manipulation of $\mathrm{PeTCO}_{2}$ under iso-oxic conditions. In this way, the $\mathrm{CBF}$ changes that result from changes in $\mathrm{PaCO}_{2}$ are not adversely effected by the unaccounted changes in $\mathrm{PaO}_{2}$. They also enable various patterns of gas challenges with transitions such as a step change, ramp, or multi-block design that can be tailored to the patient population and permit statistically rigorous assessment of $\operatorname{CVR}(24,25,71,78)$. The prospective targeting method includes end-inspiratory rebreathing assuring the equality of the $\mathrm{PeTCO}_{2}$ and $\mathrm{PaCO}_{2}(79,80)$ and providing the same vasoactive stimulus in comparative and longitudinal studies; an important requirement that can not be met by other techniques previously described here (26) (an example of prospective end-tidal targeting is provided in Figure 3). Prospective targeting is safe (all inspired gases contain $\mathrm{O}_{2}$ ) and well tolerated and requires only a minimal amount of cooperation on the part of study subjects. Using this technique, children as young as 10 years of age with cerebrovascular disease (81) and as young as 13 years of age with concussion (17) have undergone successful CVR imaging. Most importantly, the rigorous control of end-tidal gases permitted by this technique translates into precise voxel tracking during BOLD image acquisition and highly reliable and reproducible between (22) and within-subject measures of CVR $(26,73)$. This level of performance however comes with

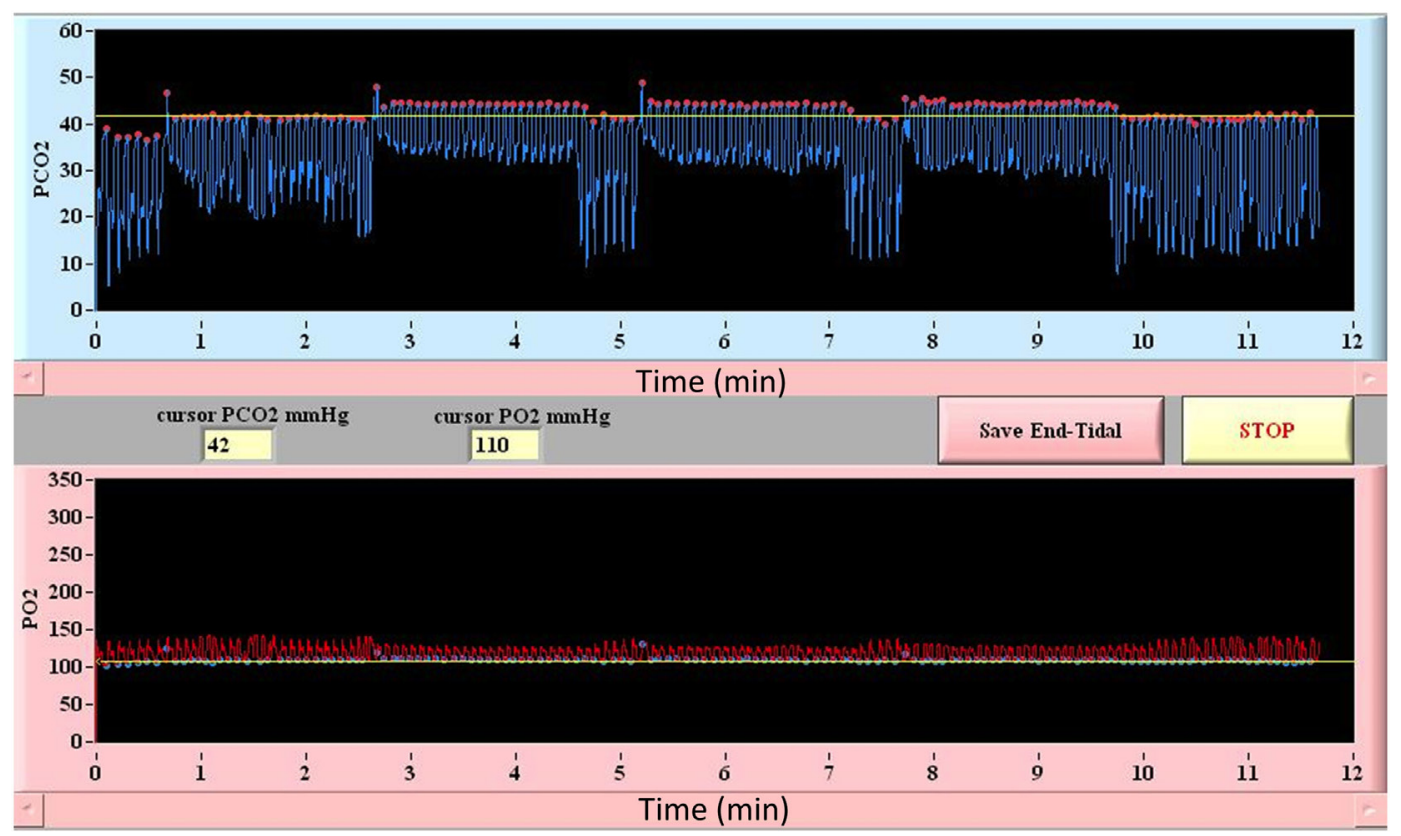

FIGURE 3 | Block design breathing protocol using model-based prospective end-tidal $\mathrm{ETCO}_{2}$ and $\mathrm{ETO}_{2}$ targeting. Triple hypercapnic stimulus during controlled iso-oxic conditions is illustrated. Breath-by-breath confirmation of $\mathrm{ETCO}_{2}$ and $\mathrm{ETO}_{2}$ allows for accurate measurement and interpretation of $\mathrm{CVR}$ assessments. 

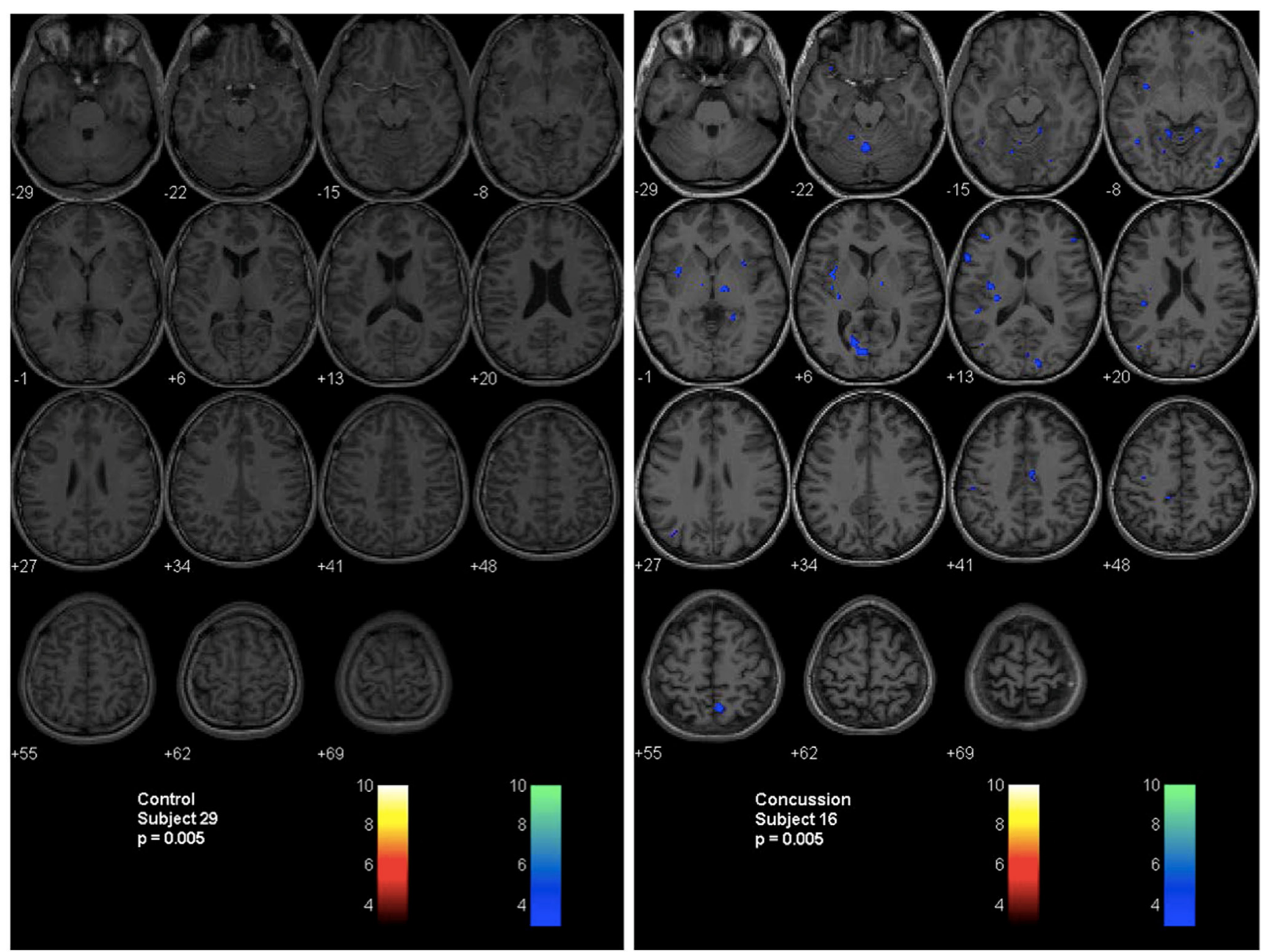

FIGURE 4 | Second-level analysis maps and postconcussion symptom scale scores for healthy control subject and adolescent postconcussion syndrome patient. Second level individual comparisons examined at the $p=0.005$ level demonstrate no evidence of abnormal voxels in the healthy control subject compared to the atlas of normal controls (left panel). Quantitative patient-specific alterations in cerebrovascular responsiveness are demonstrated in the adolescent postconcussion syndrome patient (right panel).

some disadvantages compared to other techniques including the need for specialized equipment including the breathing circuits, gas blender, and customized gases as well as the need for on-site personnel that have experience in respiratory physiology and mechanics and are trained to operate the equipment.

When compared, both inhaled $\mathrm{CO}_{2}$ and breath-holding have been found to provide an inferior assessment of absolute CVR compared to prospective targeting (82). Although associated with an increased investment in cost and personnel, prospective targeting is the only available technique capable of providing an accurate, reliable, and reproducible vasoactive stimulus and thus optimal assessment of CVR.

\section{Data Analysis}

The primary goal ofCVR data analysis is to assess whether global or regional differences in CVR observed among a patient or a group of patients are significantly different compared to those observed among healthy control subjects and that the characteristics of the disease under investigation are responsible for this difference. Because of the wide variability in structural brain characteristics and CVR values that can occur in healthy subjects, it is important that CVR data analysis techniques provide a standardized method of subject comparison that control for these subtleties. Accomplishing this aim requires the generation of a sizeable normal control atlas that can account for the immaterial variables that can potentially contribute to changes in CVR including age, sex, hemoglobin concentration, physical fitness level, time of day, hormone levels, state of mind, and technical factors related to the vasoactive stimulus and MRI equipment (25). Importantly, as it relates to concussion, it is also necessary that the control atlas include patients with a history of previous but recovered concussion so that observed changes in CVR can be reliably attributed to the most recent and symptomatic concussion and not just the presence of any lifetime concussion. At present, there are several CVR data analysis techniques that can be used to facilitate these comparisons. 

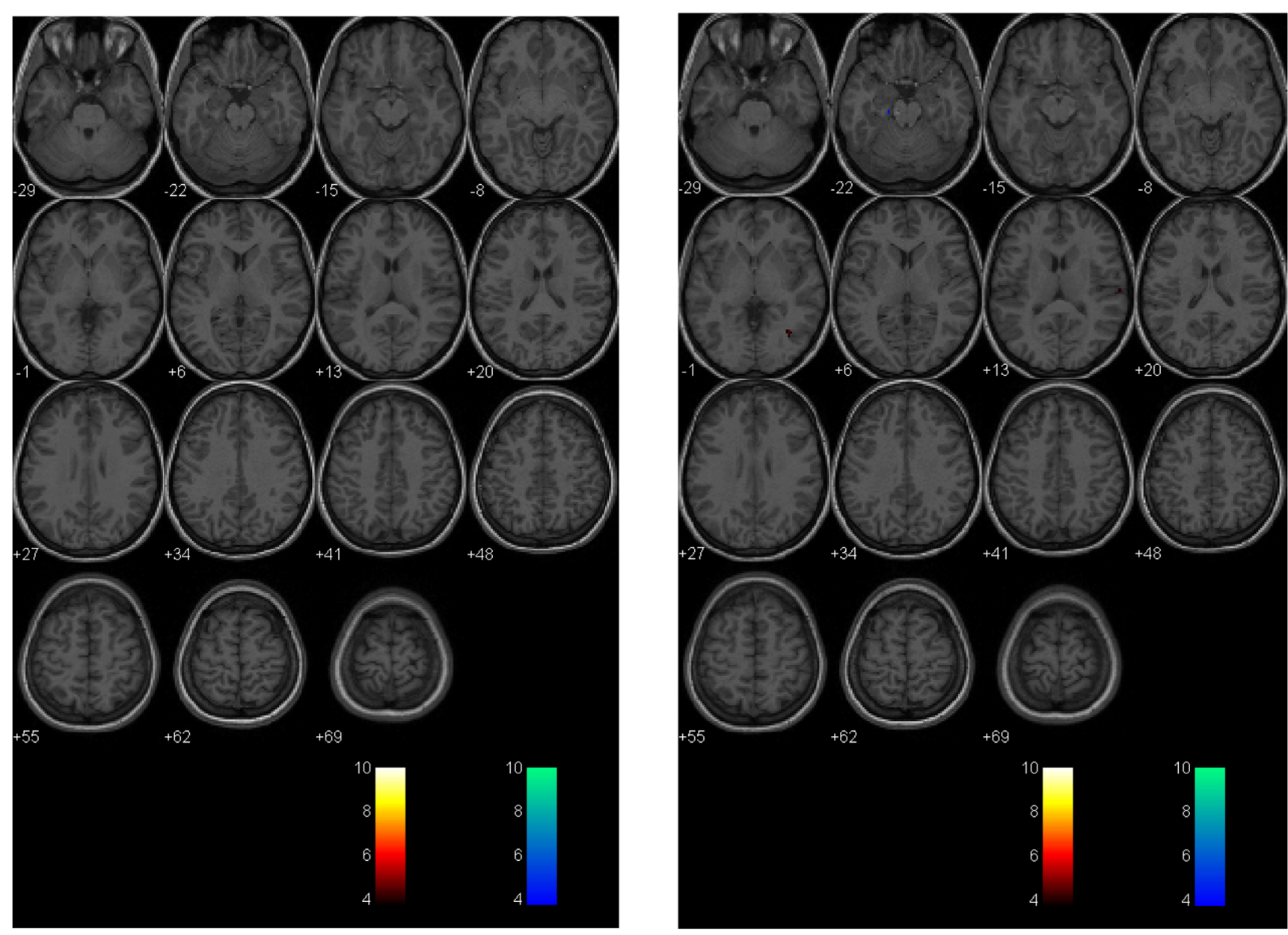

FIGURE 5 | Longitudinal assessment of healthy control subject. Second-level analysis in a healthy adolescent imaged 18 months apart and compared to a normal atlas reveals stable CVR assessment. The $p$-value is 0.005 as in Figure 4.

A detailed description of the application of such a technique has been described by Sobczyk et al. (25). A reference atlas is constructed using Analysis of Functional NeuroImages (AFNI) software to co-register each of the individual T1-weighted anatomical images into a common Montreal Neurological Institute 152 space (MNI152) using a calculated transformation subsequently applied to the BOLD data. In order to generate a CVR reference atlas, each healthy subject's anatomical and BOLD data (whether normal control or patient) are normalized into the same MNI152 space and time aligned with $\mathrm{PeTCO}_{2}$ allowing CVR to be calculated for each voxel. The mean $(\mathrm{M}) \pm \mathrm{SD}$ of CVR for each voxel location is calculated. In a test scan, the CVR of the corresponding voxel is scored according to its $z$ score $[z=(M-\mathrm{CVR}) /$ $\mathrm{SD}$ ] by comparison to the reference atlas. This normalizes the voxel CVR score for anatomical location and provides a probability score of its being within the normal range. The magnitude of the $z$ score (larger numbers implying low probabilities of being normal) is color-coded and superimposed onto the subject's anatomic scan providing a comprehensive view of the distribution of normal and abnormal responses in the scan. This CVR scoring technique therefore takes into account the voxel's anatomical location as well as the normal variability. It would be particularly important for the study of concussion, where the injury may be diffuse or isolated in particular anatomical regions.

A second technique described by Mutch et al. $(14,17,71)$ uses a similar methodology. In this technique, anatomical and BOLD data from the normal control subjects are post-processed using SPM, normalized into MNI152 space and time-aligned with the $\mathrm{PETCO}_{2}$ output to generate a normal CVR atlas. To assess voxelby-voxel differences in the CVR response between an individual subject (control or patient), second-level analyses can be completed under conditions that examine the number of voxels that are greater than or less than those observed in the control atlas with different levels of statistical significance. In this way, voxelby-voxel differences can be color coded and overlaid onto the anatomical maps to provide a qualitative assessment of regional CVR abnormalities (Figure 4). More importantly, quantitative biomarkers for voxels greater than, less than, or the sum of these (total abnormal voxel counts) can be generated and compared to other objective measures of the condition under investigation. 

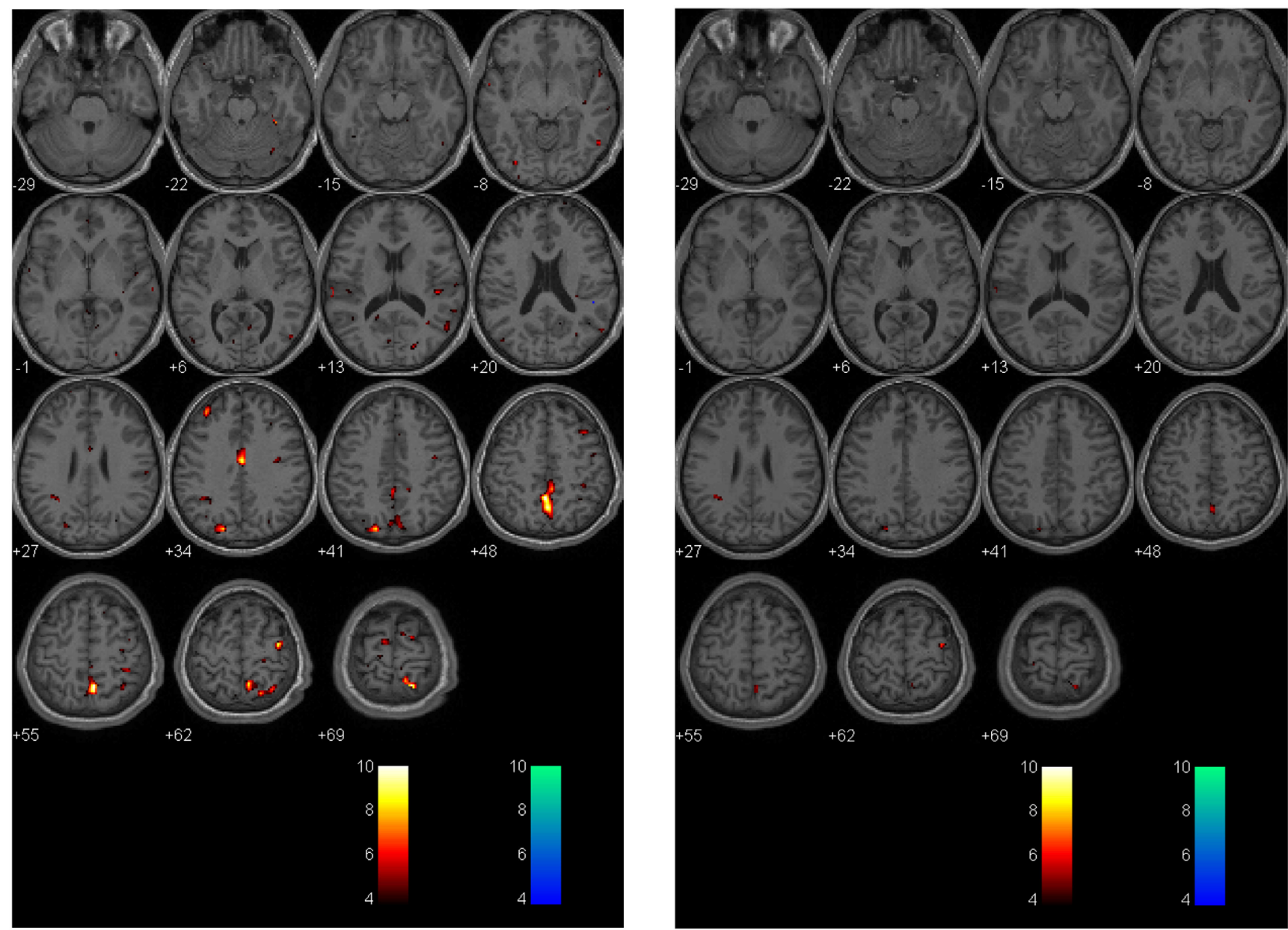

FIGURE 6 | Longitudinal assessment of adolescent postconcussion syndrome patient. Symptomatic adolescent PCS patient imaged following abnormal formal neuropsychological testing and symptom-limiting threshold on graded aerobic treadmill testing 3 months post-injury [left panel; image reproduced with permission from Journal of Neurosurgery [Mutch et al. (17)]. Patient re-imaged 5 months later following treatment with sub-maximal exercise prescription resulting in symptom, neuropsychological, and physiological recovery (right panel). The $p$-value is 0.005 as in Figures 4 and $\mathbf{5}$.

Similar to the $z$-scores described above, these quantitative biomarkers can be used to longitudinally track CVR abnormalities over time in individual subjects or patients (Figures 5 and 6).

In addition to evaluating global and regional voxel-wise differences in CVR between normal control subjects and patients, additional modifications to these techniques can be used to measure the rate of CBF increase to a controlled vasoactive stimulus. Using the prospective targeting approach for $\mathrm{CO}_{2}$ delivery, subjects undergo an abrupt (within two breaths) step change in $\mathrm{PeTCO}_{2}$ during BOLD imaging acquisition (24). Analysis can then proceed by one of two methods. First, both the steady state CVR and the rate of change of the BOLD response (expressed as a time constant of change for an exponential function or tau) can be calculated on a voxel-by-voxel basis. These measurements can be color-coded similar to the raw CVR data and overlaid onto the subject's anatomical maps to generate tau and steady state CVR maps that can be analyzed in a fashion analogous to the $z$-map technique (24). Second, transfer function analysis can also be applied, which provides even greater information about the dynamic BOLD response to hypercapnia including gain, coherence, and phase (78). Preliminary studies suggest that the onset and time course of the CBF response to a vasodilatory stimulus is prolonged in patients with other cerebrovascular diseases such as steno-occlusive disease and Alzheimer's disease $(24,78)$. To date, there have been no studies that have examined the dynamic BOLD response to a vasodilatory stimulus in TBI or concussion.

\section{OVERVIEW OF CVR STUDIES IN CONCUSSION}

The preceding discussion of cerebrovascular pathophysiology in TBI, and CVR measurement techniques, provides a perspective from which to evaluate the quality and applicability of previous (and future) studies that examine CVR in patients with concussion (Table 2).

We searched for published studies that met the following criteria: (1) included a study population of one or more patients with concussion or mTBI and (2) utilized MRI-based techniques to measure CVR or cerebrovascular responsiveness. Overall, four 
TABLE 2 | Methodological framework for evaluation of cerebrovascular reactivity measurement in concussion.

\section{Questions}

(1) What is the patient population being investigated (e.g., acute concussion, sports-related concussion, postconcussion syndrome, $\mathrm{mTBl})$ ?

(2) What is the study methodology (e.g., cross-sectional and longitudinal)?

(3) What clinical measures are used to assess concussion patients (e.g., symptom inventory, neurocognitive tests)?

(4) Are the control group subjects appropriate for comparison to the patient population?

(5) What neuroimaging sequences are used to assess cerebral blood flow (e.g., BOLD MRI, and ASL)?

(6) What vasoactive stimulus is used to manipulate cerebral blood flow?

(7) Is the magnitude of the vasoactive stimulus measured and reported?

(8) Is the magnitude of the vasoactive stimulus equal between groups and within subjects?

(9) What are the results of CVR measurement (e.g., group differences and individual differences)?

(10) Does the CVR assessment technique yield any quantitative biomarkers?

(11) What is the relationship between the CVR results and other neuroimaging findings?

mTBI, mild traumatic brain injury; BOLD, blood oxygen-dependent level; ASL, arterial spin labeling; CVR, cerebrovascular reactivity.

studies met the inclusion criteria and were analyzed (results summarized in Table 3).

Among these, Mutch et al. (14) utilized prospective iso-oxic targeting of $\mathrm{PETCO}_{2}$ and BOLD MRI to examine cerebrovascular responsiveness in adult concussion patients and normal control subjects. In this pilot study, they observed no differences in $\mathrm{PETCO}_{2}$ changes and whole brain CVR between subject groups. However, they demonstrated quantified patient-specific alterations in CVR that were present in both symptomatic and asymptomatic concussion patients that were absent among healthy control subjects. Chan et al. (15) performed a longitudinal study on a single mTBI patient during the symptomatic and asymptomatic stages of mTBI. Using breath-holding during BOLD MRI, they observed qualitative asymmetries in whole brain CVR on the initial assessment that were less asymmetrical on follow-up assessment. Miltana et al. (16) used inhaled $\mathrm{CO}_{2}$ during BOLD MRI to evaluate CVR in a group of acute SRC patients and healthy control subjects. They observed CVR values within certain regions of interest that were higher in the SRC group compared to the control group. Increased functional connectivity within the hippocampus correlated with increased CVR values. No group differences were observed in resting mean CBF measured with pCASL. Finally, Mutch et al. (17) utilized prospective $\mathrm{PeTCO}_{2}$ and BOLD MRI to examine cerebrovascular responsiveness in adolescent PCS patients and normal controls. With a standardized $\mathrm{PeTCO}_{2}$, there was a reduced cerebrovascular responsiveness observed in the PCS group. Patient-specific qualitative and quantitative alterations in cerebrovascular responsiveness were observed in all individual PCS patients. Preliminary receiver operator curve analysis revealed an area under the curve of $0.87(p<0.0001)$ for voxels manifesting a CVR response greater than the control response and 0.80 $(p=0.0003)$ for those manifesting a CVR response lower than the control response. No differences in global mean CBF measured with pCASL were observed; however, patient-specific alterations in regional resting $\mathrm{CBF}$ were observed among PCS patients.

\section{COMMENTS}

Although recent neuroimaging studies have advanced our understanding of concussion and mTBI, a diagnostic assessment tool that provides qualitative and quantitative assessment of brain pathophysiology in this clinical population has remained elusive. Despite the increasing popularity and application of neuroimaging tools such as diffusion tensor imaging, task-based and resting state functional MRI, and MRI-based assessment of resting CBF in the academic literature, none of these techniques are presently capable of providing clinically meaningful information that impacts the management of individual concussion patients (83, 84). However, in its relatively short history, MRI-based CVR imaging has the potential to play an instrumental role in the diagnosis, prognostication, and management of several cerebrovascular disorders. Because TBI and concussion are thought to be associated with alterations in cellular metabolism and CBF that are similar to those observed in stroke, the emerging concept of concussion as a form of cerebrovascular disease is novel and intriguing. Consequently, there is hope that MRI-based CBF and CVR studies will not only uncover the pathophysiological mechanisms governing individual concussion symptoms but may also provide a means to accurately and reliably contribute to the diagnosis, classification, prognostication, and confirmed recovery in individual acute concussion and PCS patients.

As future studies venture into this evolving landscape, it is important for clinicians and researchers to have a thorough appreciation of the role of CBF and CVR in TBI, and have an understanding of the methodological limitations of the neuroimaging assessment tools that are used to investigate these processes. As discussed here, currently available MRI sequences provide an accurate, reliable, and reproducible measure of $\mathrm{CBF}$. Unfortunately, the vasoactive stimuli used to manipulate $\mathrm{CBF}$, and therefore, permit accurate measurement of CVR are not methodologically equal. Readers are reminded that in order to provide accurate, reliable, and reproducible measures of CVR that the magnitude of the vasoactive stimulus must be measurable and equal across subjects, otherwise conclusions regarding the effects of a disease process on cerebrovascular physiology can not be reliably made. This issue is sufficient to account for the paucity of reliable and comparable data regarding the role of cerebrovascular dysfunction in concussion, to date.

For MRI-based CVR assessment to impact the management of individual concussion patients, a number of unique challenges must be overcome. Unlike cerebrovascular diseases such as stroke which have well validated neuroimaging and clinical outcome measures against which CVR findings can be compared, the severity of concussion and its effect on subjective physical, cognitive, and emotional functioning are not easily measured. Many of the symptoms of concussion are non-specific and found in other conditions such as primary headache disorders, whiplash, depression, and endorsed among normal subjects in the absence of TBI (85-90). Although neurocognitive and neuropsychiatric tools can provide an objective measure of some of these processes, 


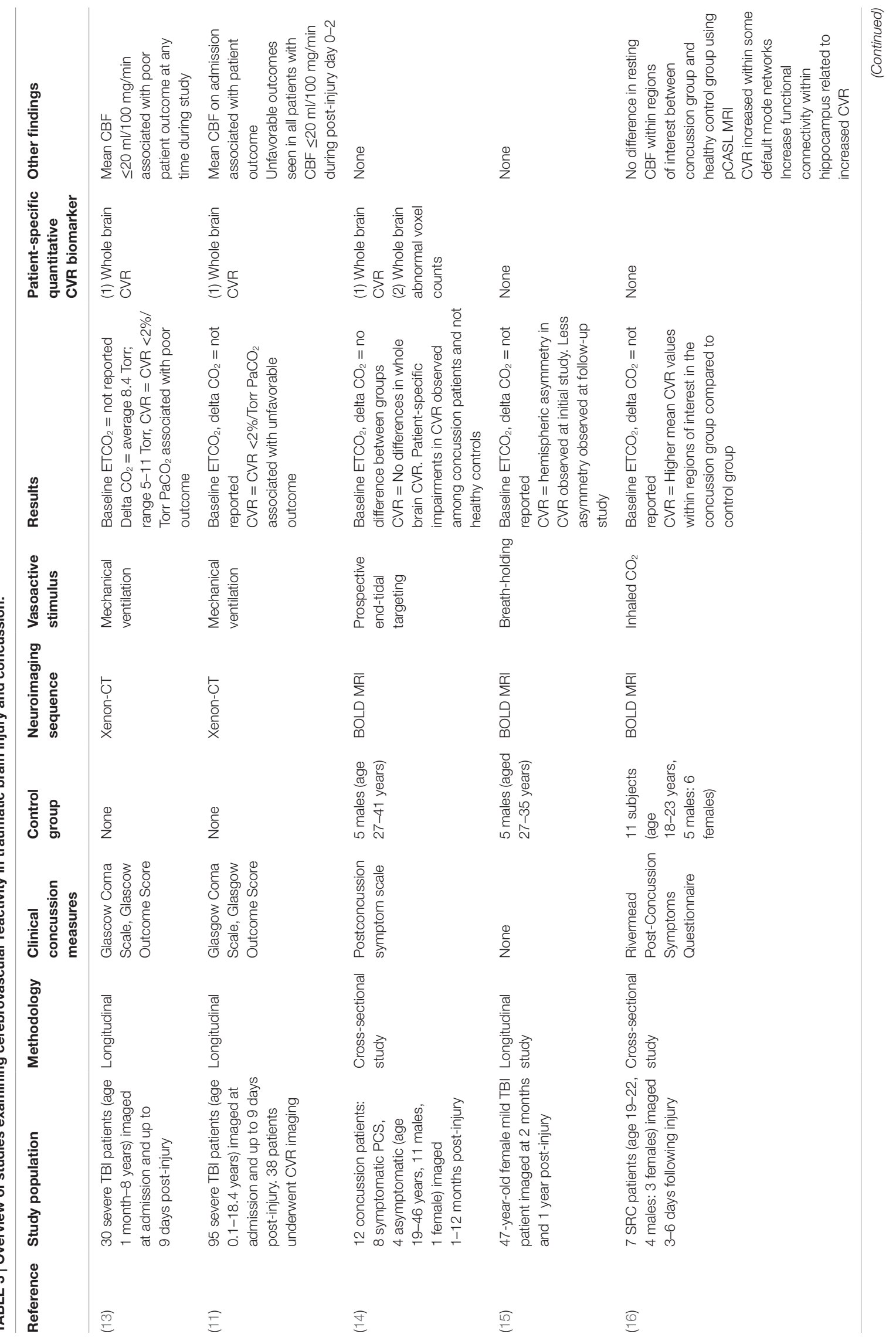




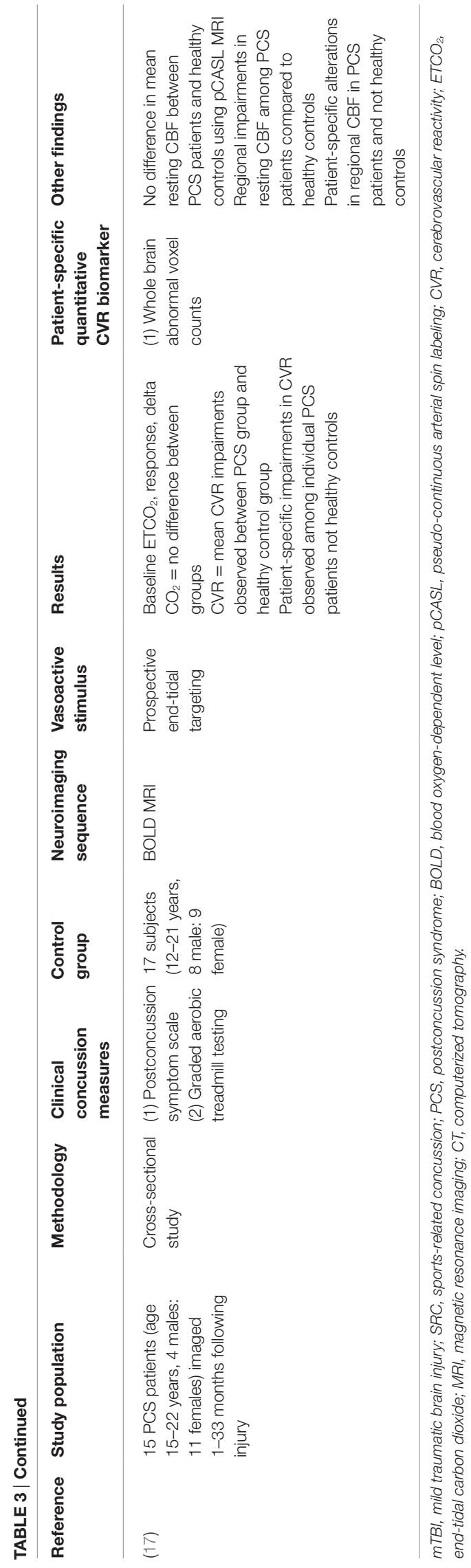

there remain a significant proportion of concussion patients in whom these studies are normal despite ongoing symptoms. Not only does this reinforce the need to apply a multi-disciplinary approach to concussion diagnosis and recovery assessment but also suggests that neuroimaging studies that generate potential quantitative biomarkers that rely on an association with scores based on other objective tools such as these must be interpreted with caution and may not be generalizable to broader acute concussion and PCS populations. Most importantly, because patients with concussion may have pre-existing conditions or develop neurological complications or disorders such as depression, migraine headaches, and other neurodegenerative diseases such as chronic traumatic encephalopathy that may also have a cerebrovascular etiology, the potential for using this technology as a diagnostic and medical clearance tool will rely on its ability to reliably distinguish between concussion and these pre-existing and post-injury disorders. Given the enormity of factors that influence CVR in normal healthy subjects and the likelihood that the alterations in CVR that occur in concussion are much more subtle than those that characterize other cerebrovascular disease, at present, the availability of a precise reproducible vasoactive stimulus reduces the SNR optimizing the opportunity to identify the cerebrovascular biomarkers associated with this condition.

In summary, preliminary research suggests that concussion is a heterogeneous condition that presents with patient-specific alterations in cerebrovascular physiology that can be safely and reliably assessed using validated MRI-based CBF and CVR assessment techniques. Future studies are warranted to determine the magnitude and natural history of these alterations following acute concussion, their relationship to other objective concussion assessment tools, and whether these alterations can be ameliorated through the application of targeted rehabilitation strategies.

\section{ETHICS STATEMENT}

Images included in this manuscript were obtained from a study approved by the University of Manitoba Biomedical Research Ethics Board. Informed patient and parental consent (for subjects 18 years of age and younger) was obtained for all research subjects (postconcussion patients and normal control subjects). This research did not include any vulnerable populations, persons with disabilities, or endangered animal species.

\section{AUTHOR CONTRIBUTIONS}

Conception and design of the work (all authors). Drafting the work and revising it critically for important intellectual content (all authors). Final approval of the version to be published (all authors). Agreement to be accountable for all aspects of the work ensuring that questions related to the accuracy or integrity of any part of the work are appropriately investigated and resolved (all authors).

\section{FUNDING}

Funding for this study was provided by Health Sciences Centre Research Foundation and Manitoba Health Research Council. 


\section{REFERENCES}

1. Golding EM. Sequelae following traumatic brain injury. The cerebrovascular perspective. Brain Res Brain Res Rev (2002) 38:377-88. doi:10.1016/ S0165-0173(02)00141-8

2. Marshall LF, Marshall SB, Klauber MR, Van Berkum Clark M, Eisenberg $\mathrm{H}$, Jane JA, et al. The diagnosis of head injury requires a classification based on computed axial tomography. J Neurotrauma (1992) 9(Suppl 1):S287-92.

3. Ashwal S, Babikian T, Gardner-Nichols J, Freier MC, Tong KA, Holshouser BA. Susceptibility-weighted imaging and proton magnetic resonance spectroscopy in assessment of outcome after pediatric traumatic brain injury. Arch Phys Med Rehabil (2006) 87:S50-8. doi:10.1016/j. apmr.2006.07.275

4. McCrory P, Meeuwisse WH, Aubry M, Cantu B, Dvorak J, Echemendia RJ, et al. Consensus statement on concussion in sport: the 4th International Conference on Concussion in Sport held in Zurich, November 2012. $\mathrm{Br}$ J Sports Med (2013) 47:250-8. doi:10.1136/bjsports-2013-092313

5. Ellis MJ, Leiter J, Hall T, McDonald PJ, Sawyer S, Silver N, et al. Neuroimaging findings in pediatric sports-related concussion. J Neurosurg Pediatr (2015) 16(3):241-7. doi:10.3171/2015.1.PEDS14510

6. Giza CC, Hovda DA. The neurometabolic cascade of concussion. J Athl Train (2001) 36:228-35.

7. Giza CC, Hovda DA. The new neurometabolic cascade of concussion. Neurosurgery(2014)75(Suppl4):S24-33.doi:10.1227/NEU.0000000000000505

8. Bouma GJ, Muizelaar JP, Choi SC, Newlon PG, Young HF. Cerebral circulation and metabolism after severe traumatic brain injury: the elusive role of ischemia. J Neurosurg (1991) 75:685-93. doi:10.3171/jns.1991.75.5.0685

9. Coles JP, Fryer TD, Smielewski P, Chatfield DA, Steiner LA, Johnston AJ, et al. Incidence and mechanisms of cerebral ischemia in early clinical head injury. J Cereb Blood Flow Metab (2004) 24:202-11. doi:10.1097/01. WCB.0000103022.98348.24

10. Wintermark M, van Melle G, Schnyder P, Revelly JP, Porchet F, Regli $\mathrm{L}$, et al. Admission perfusion CT: prognostic value in patients with severe head trauma. Radiology (2004) 232:211-20. doi:10.1148/ radiol.2321030824

11. Adelson PD, Srinivas R, Chang Y, Bell M, Kochanek PM. Cerebrovascular response in children following severe traumatic brain injury. Childs Nerv Syst (2011) 27:1465-76. doi:10.1007/s00381-011-1476-z

12. Fierstra J, Sobczyk O, Battisti-Charbonney A, Mandell DM, Poublanc J, Crawley AP, et al. Measuring cerebrovascular reactivity: what stimulus to use? J Physiol (2013) 591(23):5809-21. doi:10.1113/jphysiol.2013.259150

13. Adelson PD, Clyde B, Kochanek PM, Wisniewski SR, Marion DW, Yonas H. Cerebrovascular response in infants and young children following severe traumatic brain injury: a preliminary report. Pediatr Neurosurg (1997) 26:200-7. doi:10.1159/000121192

14. Mutch WA, Ellis MJ, Graham MR, Wourms V, Raban R, Fisher JA, et al. Brain MRI $\mathrm{CO}_{2}$ stress testing: a pilot study in patients with concussion. PLoS One (2014) 9:e102181. doi:10.1371/journal.pone.0102181

15. Chan ST, Evans KC, Rosen BR, Song TY, Kwong KK. A case study of magnetic resonance imaging of cerebrovascular reactivity: a powerful imaging marker for mild traumatic brain injury. Brain Inj (2015) 29:403-7. doi:10.3109/0269 9052.2014.974209

16. Militana AR, Donahue MJ, Sills AK, Solomon GS, Gregory AJ, Strother MK, et al. Alterations in default-mode network connectivity may be influenced by cerebrovascular changes within 1 week of sports related concussion in college varsity athletes: a pilot study. Brain Imaging Behav (2015). doi:10.1007/ s11682-015-9407-3

17. Mutch WA, Ellis MJ, Ryner LN, Ruth Graham M, Dufault B, Gregson $\mathrm{B}$, et al. Brain magnetic resonance imaging $\mathrm{CO}_{2}$ stress testing in adolescent postconcussion syndrome. J Neurosurg (2015):1-13. doi:10.3171/2015.6.JNS15972

18. Attwell D, Buchan AM, Charpak S, Lauritzen M, Macvicar BA, Newman EA. Glial and neuronal control of brain blood flow. Nature (2010) 468:232-43. doi:10.1038/nature09613

19. Hall CN, Reynell C, Gesslein B, Hamilton NB, Mishra A, Sutherland BA, et al. Capillary pericytes regulate cerebral blood flow in health and disease. Nature (2014) 508:55-60. doi:10.1038/nature13165
20. Stanimirovic DB, Friedman A. Pathophysiology of the neurovascular unit: disease cause or consequence? J Cereb Blood Flow Metab (2012) 32:1207-21. doi:10.1038/jcbfm.2012.25

21. Iadecola C. The pathobiology of vascular dementia. Neuron (2013) 80:844-66. doi:10.1016/j.neuron.2013.10.008

22. Sobczyk O, Battisti-Charbonney A, Fierstra J, Mandell DM, Poublanc J, Crawley AP, et al. A conceptual model for $\mathrm{CO}_{2}$-induced redistribution of cerebral blood flow with experimental confirmation using BOLD MRI. Neuroimage (2014) 92:56-68. doi:10.1016/j.neuroimage.2014.01.051

23. Faraci FM, Heistad DD. Regulation of large cerebral arteries and cerebral microvascular pressure. Circ Res (1990) 66:8-17. doi:10.1161/01.RES.66.1.8

24. Poublanc J, Crawley AP, Sobczyk O, Montandon G, Sam K, Mandell DM, et al. Measuring cerebrovascular reactivity: the dynamic response to a step hypercapnic stimulus. J Cereb Blood Flow Metab (2015) 35(11):1746-56. doi:10.1038/jcbfm.2015.114

25. Sobczyk O, Battisti-Charbonney A, Poublanc J, Crawley AP, Sam K, Fierstra J, et al. Assessing cerebrovascular reactivity abnormality by comparison to a reference atlas. J Cereb Blood Flow Metab (2015) 35:213-20. doi:10.1038/ jcbfm.2014.184

26. Sobczyk O, Crawley AP, Poublanc J, Sam K, Mandell DM, Mikulis DJ, et al. Identifying significant changes in cerebrovascular reactivity to carbon dioxide. AJNR Am J Neuroradiol (2016). doi:10.3174/ajnr.A4679

27. Kassner A, Winter JD, Poublanc J, Mikulis DJ, Crawley AP. Blood-oxygen level dependent MRI measures of cerebrovascular reactivity using a controlled respiratory challenge: reproducibility and gender differences. JMagn Reson Imaging (2010) 31:298-304. doi:10.1002/jmri.22044

28. Bhogal AA, Siero JC, Fisher JA, Froeling M, Luijten P, Philippens M, et al. Investigating the non-linearity of the BOLD cerebrovascular reactivity response to targeted hypo/hypercapnia at 7T. Neuroimage (2014) 98:296-305. doi:10.1016/j.neuroimage.2014.05.006

29. Regan RE, Fisher JA, Duffin J. Factors affecting the determination of cerebrovascular reactivity. Brain Behav (2014) 4:775-88. doi:10.1002/brb3.275

30. Bhogal AA, Philippens ME, Siero JC, Fisher JA, Petersen ET, Luijten PR, et al. Examining the regional and cerebral depth-dependent BOLD cerebrovascular reactivity response at 7T. Neuroimage (2015) 114:239-48. doi:10.1016/j. neuroimage.2015.04.014

31. Balucani C, Silvestrini M. Carotid atherosclerotic disease and cognitive function: mechanisms identifying new therapeutic targets. Int J Stroke (2011) 6:368-9. doi:10.1111/j.1747-4949.2011.00628.x

32. Fierstra J, Poublanc J, Han JS, Silver F, Tymianski M, Crawley AP, et al. Steal physiology is spatially associated with cortical thinning. J Neurol Neurosurg Psychiatry (2010) 81:290-3. doi:10.1136/jnnp.2009.188078

33. Lythgoe DJ, Williams SC, Cullinane M, Markus HS. Mapping of cerebrovascular reactivity using BOLD magnetic resonance imaging. Magn Reson Imaging (1999) 17:495-502. doi:10.1016/S0730-725X(98)00211-2

34. Kuroda S, Houkin K, Kamiyama H, Mitsumori K, Iwasaki Y, Abe H. Longterm prognosis of medically treated patients with internal carotid or middle cerebral artery occlusion: can acetazolamide test predict it? Stroke (2001) 32:2110-6. doi:10.1161/hs0901.095692

35. Mandell DM, Han JS, Poublanc J, Crawley AP, Stainsby JA, Fisher JA, et al. Mapping cerebrovascular reactivity using blood oxygen level-dependent MRI in patients with arterial steno-occlusive disease: comparison with arterial spin labeling MRI.Stroke (2008) 39:2021-8. doi:10.1161/STROKEAHA.107.506709

36. Bokkers RP, van Osch MJ, Klijn CJ, Kappelle LJ, Hendrikse J. Cerebrovascular reactivity within perfusion territories in patients with an internal carotid artery occlusion. J Neurol Neurosurg Psychiatry (2011) 82:1011-6. doi:10.1136/ jnnp.2010.233338

37. Mandell DM, Han JS, Poublanc J, Crawley AP, Fierstra J, Tymianski M, et al. Quantitative measurement of cerebrovascular reactivity by blood oxygen level-dependent MR imaging in patients with intracranial stenosis: preoperative cerebrovascular reactivity predicts the effect of extracranial-intracranial bypass surgery. AJNR Am J Neuroradiol (2011) 32:721-7. doi:10.3174/ajnr. A2365

38. Fierstra J, Conklin J, Krings T, Slessarev M, Han JS, Fisher JA, et al. Impaired peri-nidal cerebrovascular reserve in seizure patients with brain arteriovenous malformations. Brain (2011) 134:100-9. doi:10.1093/brain/awq286

39. Fierstra J, Spieth S, Tran L, Conklin J, Tymianski M, ter Brugge KG, et al. Severely impaired cerebrovascular reserve in patients with 
cerebral proliferative angiopathy. JNeurosurg Pediatr (2011) 8:310-5. doi:10.3171/2011.6.PEDS1170

40. da Costa L, Fierstra J, Fisher JA, Mikulis DJ, Han JS, Tymianski M. BOLD MRI and early impairment of cerebrovascular reserve after aneurysmal subarachnoid hemorrhage. J Magn Reson Imaging (2014) 40:972-9. doi:10.1002/ jmri.24474

41. Schoof J, Lubahn W, Baeumer M, Kross R, Wallesch CW, Kozian A, et al. Impaired cerebral autoregulation distal to carotid stenosis/occlusion is associated with increased risk of stroke at cardiac surgery with cardiopulmonary bypass. J Thorac Cardiovasc Surg (2007) 134:690-6. doi:10.1016/j. jtcvs.2007.03.018

42. Gupta A, Chazen JL, Hartman M, Delgado D, Anumula N, Shao H, et al. Cerebrovascular reserve and stroke risk in patients with carotid stenosis or occlusion: a systematic review and meta-analysis. Stroke (2012) 43:2884-91. doi:10.1161/STROKEAHA.112.663716

43. Reinhard M, Schwarzer G, Briel M, Altamura C, Palazzo P, King A, et al. Cerebrovascular reactivity predicts stroke in high-grade carotid artery disease. Neurology (2014) 83:1424-31. doi:10.1212/WNL.0000000000000888

44. Corps KN, Roth TL, McGavern DB. Inflammation and neuroprotection in traumatic brain injury. JAMA Neurol (2015) 72:355-62. doi:10.1001/ jamaneurol.2014.3558

45. Hinson HE, Rowell S, Schreiber M. Clinical evidence of inflammation driving secondary brain injury: a systematic review. J Trauma Acute Care Surg (2015) 78:184-91. doi:10.1097/TA.0000000000000468

46. Graham DI, Adams JH. Ischaemic brain damage in fatal head injuries. Lancet (1971) 1:265-6. doi:10.1016/S0140-6736(71)91003-8

47. Graham DI, Ford I, Adams JH, Doyle D, Teasdale GM, Lawrence AE, et al. Ischaemic brain damage is still common in fatal non-missile head injury. J Neurol Neurosurg Psychiatry (1989) 52:346-50. doi:10.1136/ jnnp.52.3.346

48. Marion DW, Bouma GJ. The use of stable xenon-enhanced computed tomographic studies of cerebral blood flow to define changes in cerebral carbon dioxide vasoresponsivity caused by a severe head injury. Neurosurgery (1991) 29:869-73. doi:10.1097/00006123-199112000-00011

49. Bonne O, Gilboa A, Louzoun Y, Kempf-Sherf O, Katz M, Fishman $\mathrm{Y}$, et al. Cerebral blood flow in chronic symptomatic mild traumatic brain injury. Psychiatry Res (2003) 124:141-52. doi:10.1016/ S0925-4927(03)00109-4

50. Peskind ER, Brody D, Cernak I, McKee A, Ruff RL. Military- and sports-related mild traumatic brain injury: clinical presentation, management, and longterm consequences. J Clin Psychiatry (2013) 74:180-8; quiz 188. doi:10.4088/ JCP.12011colc

51. Ahmed F, Plantman S, Cernak I, Agoston DV. The temporal pattern of changes in serum biomarker levels reveals complex and dynamically changing pathologies after exposure to a single low-intensity blast in mice. Front Neurol (2015) 6:114. doi:10.3389/fneur.2015.00114

52. Maugans TA, Farley C, Altaye M, Leach J, Cecil KM. Pediatric sports-related concussion produces cerebral blood flow alterations. Pediatrics (2012) 129:28-37. doi:10.1542/peds.2011-2083

53. Bartnik-Olson BL, Holshouser B, Wang H, Grube M, Tong K, Wong V, et al. Impaired neurovascular unit function contributes to persistent symptoms after concussion: a pilot study. J Neurotrauma (2014) 31:1497-506. doi:10.1089/ neu. 2013.3213

54. Meier TB, Bellgowan PS, Singh R, Kuplicki R, Polanski DW, Mayer AR. Recovery of cerebral blood flow following sports-related concussion. JAMA Neurol (2015) 72(5):530-8. doi:10.1001/jamaneurol.2014.4778

55. Wang Y, Nelson LD, LaRoche AA, Pfaller AY, Nencka AS, Koch KM, et al. Cerebral blood flow alterations in acute sport-related concussion. J Neurotrauma (2015). doi:10.1089/neu.2015.4072

56. Gardner AJ, Tan CO, Ainslie PN, van Donkelaar P, Stanwell P, Levi CR, et al. Cerebrovascular reactivity assessed by transcranial Doppler ultrasound in sport-related concussion: a systematic review. Br J Sports Med (2014). doi:10.1136/bjsports-2014-093901

57. Davis TL, Kwong KK, Weisskoff RM, Rosen BR. Calibrated functional MRI: mapping the dynamics of oxidative metabolism. Proc Natl Acad Sci U S A (1998) 95:1834-9. doi:10.1073/pnas.95.4.1834

58. Hoge RD, Atkinson J, Gill B, Crelier GR, Marrett S, Pike GB. Linear coupling between cerebral blood flow and oxygen consumption in activated human cortex. Proc Natl Acad Sci U S A (1999) 96:9403-8. doi:10.1073/pnas.96.16.9403
59. Attwell $\mathrm{D}$, Iadecola $\mathrm{C}$. The neural basis of functional brain imaging signals. Trends Neurosci (2002) 25:621-5. doi:10.1016/S0166-2236(02)02264-6

60. Teschemacher AG, Gourine AV, Kasparov S. A role for astrocytes in sensing the brain microenvironment and neuro-metabolic integration. Neurochem Res (2015) 40:2386-93. doi:10.1007/s11064-015-1562-9

61. Wells JA, Christie IN, Hosford PS, Huckstepp RT, Angelova PR, Vihko P, et al. A critical role for purinergic signalling in the mechanisms underlying generation of BOLD fMRI responses. J Neurosci (2015) 35:5284-92. doi:10.1523/ JNEUROSCI.3787-14.2015

62. Donahue MJ, Strother MK, Hendrikse J. Novel MRI approaches for assessing cerebral hemodynamics in ischemic cerebrovascular disease. Stroke (2012) 43:903-15. doi:10.1161/STROKEAHA.111.635995

63. Vorstrup S, Brun B, Lassen NA. Evaluation of the cerebral vasodilatory capacity by the acetazolamide test before EC-IC bypass surgery in patients with occlusion of the internal carotid artery. Stroke (1986) 17:1291-8. doi:10.1161/01.STR.17.6.1291

64. Vorstrup S, Paulson OB, Lassen NA. Cerebral blood flow in acute and chronic ischemic stroke using xenon-133 inhalation tomography. Acta Neurol Scand (1986) 74:439-51. doi:10.1111/j.1600-0404.1986.tb07869.x

65. Saito H, Ogasawara K, Suzuki T, Kuroda H, Kobayashi M, Yoshida K, et al. Adverse effects of intravenous acetazolamide administration for evaluation of cerebrovascular reactivity using brain perfusion single-photon emission computed tomography in patients with major cerebral artery steno-occlusive diseases. Neurol Med Chir (2011) 51:479-83. doi:10.2176/nmc.51.479

66. Dahl A, Russell D, Rootwelt K, Nyberg-Hansen R, Kerty E. Cerebral vasoreactivity assessed with transcranial Doppler and regional cerebral blood flow measurements. Dose, serum concentration, and time course of the response to acetazolamide. Stroke (1995) 26:2302-6. doi:10.1161/01. STR.26.12.2302

67. Grossmann WM, Koeberle B. The dose-response relationship of acetazolamide on the cerebral blood flow in normal subjects. Cerebrovasc Dis (2000) 10:65-9. doi:10.1159/000016027

68. Markus HS, Harrison MJ. Estimation of cerebrovascular reactivity using transcranial Doppler, including the use of breath-holding as the vasodilatory stimulus. Stroke (1992) 23:668-73. doi:10.1161/01.STR.23.5.668

69. Ringelstein EB, Van Eyck S, Mertens I. Evaluation of cerebral vasomotor reactivity by various vasodilating stimuli: comparison of $\mathrm{CO}_{2}$ to acetazolamide. J Cereb Blood Flow Metab (1992) 12:162-8. doi:10.1038/jcbfm.1992.20

70. Gooskens I, Schmidt EA, Czosnyka M, Piechnik SK, Smielewski P, Kirkpatrick $\mathrm{PJ}$, et al. Pressure-autoregulation, $\mathrm{CO}_{2}$ reactivity and asymmetry of haemodynamic parameters in patients with carotid artery stenotic disease. A clinical appraisal. Acta Neurochir (Wien) (2003) 145:527-32; discussion 532. doi:10.1007/s00701-003-0045-y

71. Mutch WA, Mandell DM, Fisher JA, Mikulis DJ, Crawley AP, Pucci O, et al. Approaches to brain stress testing: BOLD magnetic resonance imaging with computer-controlled delivery of carbon dioxide. PLoS One (2012) 7:e47443. doi:10.1371/journal.pone.0047443

72. Regan RE, Duffin J, Fisher JA. Instability of the middle cerebral artery blood flow in response to $\mathrm{CO}_{2}$. PLoS One (2013) 8:e70751. doi:10.1371/journal. pone. 0070751

73. Spano VR, Mandell DM, Poublanc J, Sam K, Battisti-Charbonney A, Pucci $\mathrm{O}$, et al. $\mathrm{CO}_{2}$ blood oxygen level-dependent MR mapping of cerebrovascular reserve in a clinical population: safety, tolerability, and technical feasibility. Radiology (2013) 266:592-8. doi:10.1148/radiol.12112795

74. Mark CI, Slessarev M, Ito S, Han J, Fisher JA, Pike GB. Precise control of end-tidal carbon dioxide and oxygen improves BOLD and ASL cerebrovascular reactivity measures. Magn Reson Med (2010) 64:749-56. doi:10.1002/ mrm.22405

75. Wise RG, Pattinson KT, Bulte DP, Chiarelli PA, Mayhew SD, Balanos GM, et al. Dynamic forcing of end-tidal carbon dioxide and oxygen applied to functional magnetic resonance imaging. J Cereb Blood Flow Metab (2007) 27:1521-32. doi:10.1038/sj.jcbfm.9600465

76. Prisman E, Slessarev M, Han J, Poublanc J, Mardimae A, Crawley A, et al. Comparison of the effects of independently-controlled end-tidal $\mathrm{PCO}_{2}$ and $\mathrm{PO}_{2}$ on blood oxygen level-dependent (BOLD) MRI. J Magn Reson Imaging (2008) 27:185-91. doi:10.1002/jmri.21102

77. Slessarev M, Han J, Mardimae A, Prisman E, Preiss D, Volgyesi G, et al. Prospective targeting and control of end-tidal $\mathrm{CO}_{2}$ and $\mathrm{O}_{2}$ concentrations. J Physiol (2007) 581:1207-19. doi:10.1113/jphysiol.2007.129395 
78. Duffin J, Sobczyk O, Crawley AP, Poublanc J, Mikulis DJ, Fisher JA. The dynamics of cerebrovascular reactivity shown with transfer function analysis. Neuroimage (2015) 114:207-16. doi:10.1016/j.neuroimage.2015.04.029

79. Brogan TV, Robertson HT, Lamm WJ, Souders JE, Swenson ER. Carbon dioxide added late in inspiration reduces ventilation-perfusion heterogeneity without causing respiratory acidosis. J Appl Physiol (1985) (2004) 96:1894-8. doi:10.1152/japplphysiol.00160.2003

80. Ito S, Mardimae A, Han J, Duffin J, Wells G, Fedorko L, et al. Non-invasive prospective targeting of arterial $\mathrm{PCO}_{2}$ in subjects at rest. J Physiol (2008) 586:3675-82. doi:10.1113/jphysiol.2008.154716

81. Han JS, Mandell DM, Poublanc J, Mardimae A, Slessarev M, Jaigobin C, et al. BOLD-MRI cerebrovascular reactivity findings in cocaine-induced cerebral vasculitis. Nat Clin Pract Neurol (2008) 4:628-32. doi:10.1038/ ncpneuro0918

82. Tancredi FB, Hoge RD. Comparison of cerebral vascular reactivity measures obtained using breath-holding and $\mathrm{CO}_{2}$ inhalation. J Cereb Blood Flow Metab (2013) 33:1066-74. doi:10.1038/jcbfm.2013.48

83. Ellis MJ, Figley CR, Mutch WA, Massicotte E, Mikulis DJ, Essig M, et al. Neuroimaging in sports-related concussion management:current status and future directions. Curr Res Concussion (2014) 1:33-9.

84. Yuh EL, Hawryluk GW, Manley GT. Imaging concussion: a review. Neurosurgery (2014) 75(Suppl 4):S50-63. doi:10.1227/NEU.0000000000000491

85. Iverson GL. Misdiagnosis of the persistent postconcussion syndrome in patients with depression. Arch Clin Neuropsychol (2006) 21:303-10. doi:10.1016/j.acn.2005.12.008

86. Wang Y, Chan RC, Deng Y. Examination of postconcussion-like symptoms in healthy university students: relationships to subjective and objective neuropsychological function performance. Arch Clin Neuropsychol (2006) 21:339-47. doi:10.1016/j.acn.2006.03.006
87. Seifert TD. Sports concussion and associated post-traumatic headache. Headache (2013) 53:726-36. doi:10.1111/head.12087

88. Ellis MJ, Leddy JJ, Willer B. Physiological, vestibulo-ocular and cervicogenic post-concussion disorders: an evidence-based classification system with directions for treatment. Brain Inj (2014):1-11. doi:10.3109/02699052. 2014.965207

89. Brooks BL, Iverson GL, Atkins JE, Zafonte R, Berkner PD. Sex differences and self-reported attention problems during baseline concussion testing. Appl Neuropsychol Child (2015):1-8. doi:10.1080/21622965.20 14.1003066

90. Iverson GL, Silverberg ND, Mannix R, Maxwell BA, Atkins JE, Zafonte $\mathrm{R}$, et al. Factors associated with concussion-like symptom reporting in high school athletes. JAMA Pediatr (2015) 169(12):1132-40. doi:10.1001/ jamapediatrics.2015.2374

Conflict of Interest Statement: The authors or their affiliated institutions have not received any payment or services from a third party for any aspect of the submitted work. DM, JF, and JD are senior scientists at Thornhill Research Inc. (TRI), a company affiliated with the University Health Network and University of Toronto that developed the RespirAct, a patented, non-commercial research tool assembled by TRI to enable cerebrovascular reactivity studies.

Copyright (c) 2016 Ellis, Ryner, Sobczyk, Fierstra, Mikulis, Fisher, Duffin and Mutch. This is an open-access article distributed under the terms of the Creative Commons Attribution License (CC BY). The use, distribution or reproduction in other forums is permitted, provided the original author(s) or licensor are credited and that the original publication in this journal is cited, in accordance with accepted academic practice. No use, distribution or reproduction is permitted which does not comply with these terms. 